\title{
Le substrat professionnel dans Wall Street, le film d'Oliver Stone
}

\section{Michel Van der Yeught}

\section{(2) OpenEdition}

\section{Journals}

Édition électronique

URL : http://journals.openedition.org/asp/848

DOI : $10.4000 /$ asp. 848

ISBN : 978-2-8218-0398-5

ISSN : 2108-6354

\section{Éditeur}

Groupe d'étude et de recherche en anglais de spécialité

\section{Édition imprimée}

Date de publication : 1 décembre 2004

Pagination : 21-42

ISSN : 1246-8185

\section{Référence électronique}

Michel Van der Yeught, « Le substrat professionnel dans Wall Street, le film d'Oliver Stone », ASp [En ligne], 45-46 | 2004, mis en ligne le 02 mars 2010, consulté le 03 mai 2019. URL : http:// journals.openedition.org/asp/848; DOI : 10.4000/asp.848

Ce document a été généré automatiquement le 3 mai 2019.

Tous droits réservés 


\title{
Le substrat professionnel dans Wall Street, le film d'Oliver Stone
}

\author{
Michel Van der Yeught
}

\section{Introduction}

1 Les formations économiques et financières se multiplient en France et leur croissance pousse les enseignants d'anglais à proposer à leurs étudiants des documents pédagogiques audio-visuels adaptés et diversifiés comme par exemple des ouvrages de fiction à substrat professionnel (FASP). Paradoxalement, ils trouvent souvent dans des récits inventés une matière bien adaptée au travail pédagogique et propre à illustrer avec clarté des développements économiques réels (Petit 1999). Néanmoins, l'œuvre de FASP doit être doublement convaincante : non seulement l'histoire doit plaire et intéresser en tant que narration dramatisée, mais elle doit également intégrer les caractéristiques techniques des métiers mis en scène. Ces deux exigences, souvent antithétiques, sont rarement satisfaites, notamment dans le domaine financier, comme en témoigne la médiocrité de Trader (1998) et des Initiés (1999) ${ }^{1}$, deux films récents sur le monde de la bourse. Les intrigues ne présentent guère d'intérêt et sont superficiellement situées dans un monde professionnel à peine esquissé.

2 La rareté des bons films de fiction à substrat professionnel financier souligne les mérites durables du Wall Street d'Oliver Stone. Ce long métrage produit en 1987 par la Fox accuse son âge sans être devenu obsolète. Aussi longtemps que le New York Stock Exchange (NYSE) gardera son parquet et ses opérateurs en chair et en os, le film reflètera avec pertinence le fonctionnement et l'atmosphère de la grande bourse new-yorkaise. L'intrigue est un peu convenue, mais, servie par des acteurs et un metteur en scène talentueux, elle capte l'attention sans la laisser se relâcher².

3 Le plus grand mérite de l'œuvre réside cependant dans le brio et la vraisemblance avec lesquels elle entrelace la logique du récit et celle des marchés financiers. Si l'on veut bien concéder que toute fiction se nourrit d'une certaine distorsion de la réalité et exagère les 
traits des personnages, on ne peut qu'admirer en contrepartie la quantité et la qualité des éléments réalistes qui enracinent l'intrigue dans un monde professionnel, certes typifié, mais très authentique. L'extrême souci du détail technique vient sans doute du fait qu'Oliver Stone a réalisé son film en hommage à « Louis Stone, stockbroker, 1910-1985 », son père, courtier à Wall Street, ainsi que le rappelle le générique. Même vingt ans plus tard, la qualité de cette mise en contexte continue d'imposer Wall Street comme une œuvre de FASP réussie et la canonise progressivement aux États-Unis en film-culte pour boursiers en herbe ${ }^{3}$. L'œuvre reste donc essentielle pour comprendre le monde de Wall Street et très précieuse pour l'enseignement de l'anglais financier.

La richesse exceptionnelle de son substrat professionnel rend Wall Street délicat à utiliser à des fins linguistiques devant des étudiants de premier cycle. En revanche, la complexité des situations techniques, la densité de la culture professionnelle ambiante et la richesse de la langue qui en découle lui donnent des atouts incomparables face à un public plus mûr. Wall Street intéressera surtout des étudiants en quête d'un contenu solide, soucieux de s'initier au milieu boursier américain et à sa langue. Pour eux et pour leurs enseignants, cet article se propose d'en analyser le substrat professionnel qui, à notre connaissance, n'a pas encore été étudié de façon approfondie. À la suite d'un résumé du film, les différents aspects abordés seront les suivants: les lieux, les métiers, les opérations boursières, les institutions, les aspects civilisationnels, la langue et la traduction en français.

\section{Résumé du film}

5 Fils d'un modeste technicien d'aviation, le jeune Bud Fox travaille en tant que courtier chez Jackson Steinem, une maison de titres de Manhattan. Passant outre les réticences de son père vis-à-vis de Wall Street, il aspire, comme tous les yuppies ${ }^{4}$ des années 1980, à devenir riche et il rêve de travailler pour "Gekko le Grand", l'un des prédateurs d'entreprise (raider) les plus agressifs de la place. Après plusieurs tentatives infructueuses, il parvient à rencontrer son idole, et, sous sa direction, il s'initie à la spéculation. Grâce à des opérations de plus en plus lucratives (et de moins en moins honnêtes), il séduit une ex-conquête de Gekko, la décoratrice Darien Taylor, et il s'installe avec elle dans un appartement du Upper East Side qu'elle réaménage de façon somptueuse. L'ascension financière et sociale du héros se voit contrariée lorsque son mentor lance une OPA hostile sur la compagnie Bluestar Airlines où son père est employé. Le démantèlement annoncé de la société contraint Bud à prendre parti pour son père et à retourner contre Gekko les techniques boursières agressives qu'il a apprises de lui. Entretemps, le gendarme des bourses américaines, la Securities and Exchange Commission (SEC), a lancé une enquête sur les pratiques frauduleuses du grand opérateur et de son élève. Bud subit une arrestation humiliante devant ses collègues de travail et il dénonce Gekko aux autorités afin d'obtenir une réduction de sa peine de prison. Le film se conclut sur la suggestion que l'innocence, perdue à Wall Street, ne peut se retrouver que loin d'elle ${ }^{5}$.

\section{Les lieux}

$6 \quad$ Wall Street se déroule pour l'essentiel dans le quartier financier de New York mais ne cède pas à la tentation de la carte postale. La façade corinthienne du New York Stock Exchange (NYSE) est fugacement suggérée en début de film par un portique à colonnes et les lieux 
sont essentiellement traités dans une perspective professionnelle. La séquence d'ouverture montre les commuters en train de converger de bon matin vers le BasManhattan signalé de loin par le double totem du World Trade Center qui dominait le district à l'époque. Ils se pressent dans les trains et le métro ou empruntent le ferry qui se dirige vers le Staten Island Terminal ou la voie rapide surélevée (elevated highway) qui longe l'East River en face de Brooklyn.

7 La salle des marchés (trading room) de la maison de titres où travaille Bud présente une activité fébrile ainsi que des éléments techniques caractéristiques des marchés des valeurs : terminal de cotation (Quotron screen), télescripteur électronique (electronic stock ticker) où passent le code des sociétés (ticker symbol) et le cours de leurs actions (stock price ) encore cotées en fractions de dollars $(1 / 2,3 / 8,7 / 8)^{6}$. Les traders appellent leurs clients en pratiquant le cold calling, c'est-à-dire «l'appel à froid " (sans rendez-vous) afin de leur proposer des actions ou des obligations à acheter. On voit ainsi Bud répéter inlassablement les mêmes phrases commerciales destinées à convaincre ses clients.

8 Le parquet du NYSE apparaît plusieurs fois au cours du film et les quelques séquences tournées dans la salle principale (Main Room) permettent de comprendre le fonctionnement du marché. La cloche légendaire (bell) ouvre et ferme les séances. Les courtiers reçoivent leurs ordres de vente ou d'achat de l'extérieur, par exemple de la part de Bud chez Jackson Steinem, et viennent les faire exécuter à des postes de négociation ( trading post) en forme de double hémicycle répartis sur le parquet. Chacun d'entre eux est réservé à un secteur du marché (automobile, pétrole, industrie lourde, informatique, banque, etc.). Ils sont animés par des spécialistes (specialists) responsables des titres qui y sont cotés. L'agitation frénétique qui y règne en période de fluctuation des cours suscite des bousculades pittoresques dont le témoignage est d'autant plus précieux qu'elles sont en voie de disparition. Le NYSE et l'American Stock Exchange (la deuxième bourse de New York) sont les derniers marchés occidentaux à fonctionner ainsi à la criée (open outcry) selon le principe des enchères (auction trading). La quasi-totalité des autres bourses, notamment en Europe, sont informatisées et donc totalement déshumanisées.

9 Les bâtiments où se déroule l'activité boursière sont eux-mêmes situés dans une géographie fortement marquée par la hiérarchie des valeurs de Wall Street. Certains lieux sont symboliquement valorisés, d'autres fortement dévalorisés. C'est ainsi que Bud préfère vivre à Manhattan ( I I gotta live in Manhattan to be a player, Dad ») plutôt que dans le quartier populaire du Queens avec ses parents [2]. Pourtant, il habite le Upper West Side (où logeaient les misérables Porto-Ricains du film West Side Story) alors que le quartier chic recherché par les yuppies est le Upper East Side (vers la $5^{\mathrm{e}}$ Avenue) [11]. Quand il rencontre la séduisante Darien Taylor, il lui avoue qu'il vit dans le West Side. Mais devant sa moue dégoûtée, il ajoute précipitamment qu'il doit bientôt déménager vers le East Side [8]. La belle consent alors à l'écouter.

De même, les riches résident sur Long Island. Gekko a une villa (beach house) à Bridgehampton ${ }^{7}$ dans la région huppée des Hamptons, à l'est de l'île. L'ancien camarade d'université de Bud, l'avocat très WASP ${ }^{8}$, Roger Barnes, habite Oyster Bay, sur la côte nord-ouest de Long Island qui fait face au Connecticut [9]. À l'inverse, Wildwood dans le New Jersey (au sud d'Atlantic City) fait figure de trou perdu [8].

Loin de ces oasis d'opulence, la ville industrielle d'Erié en Pennsylvanie évoquée dans les dialogues [6], les hangars où le père de Bud répare les avions [10], le bar de banlieue où son fils le retrouve après le travail [2], semblent appartenir à une autre Amérique, celle 
des classes moyennes et populaires souverainement méprisées par les plus fortunés. La coupure entre ces deux mondes illustre l'opposition durable entre Wall Street et Main Street (voir section 5).

\section{Les métiers}

\subsection{La hiérarchie et la diversité des métiers}

Wall Street présente une galerie très fournie de la plupart des métiers de la bourse. Il pèse sur eux le même type de hiérarchie que sur les lieux. Au bas de l'échelle professionnelle se trouvent les coursiers (runners) qui transmettent entre les opérateurs les messages et les ordres d'opération rédigés sur papier. Survivances d'un XIX siècle d'avant le téléphone, ils ont désormais largement disparu. Au-dessus d'eux opèrent les courtiers ( brokers) qui servent d'intermédiaires entre les clients et le marché et qui sont payés à la commission. Ils ne se font guère d'illusion sur leur importance : «[...] it's big game hunters that bag the elephant, not guys like us » dit Marvin, le collègue de Bud [2]. Et au cas où ils s'en feraient, Natalie, la secrétaire du tout-puissant Gordon Gekko précise : « Mr Fox, I told you before, I'm sure you're a good broker, but our traders deal with the brokers, Mr Gekko only deals with investment bankers» [2]. Les courtiers qui négocient sur le parquet sont encore souvent appelés «courtiers à deux dollars " (two-dollar brokers) parce qu'à l'origine ils gagnaient deux dollars par exécution. Au-dessus des courtiers, se trouvent les traders qui opèrent pour leur propre compte [7]. Ils prennent plus de risques que les courtiers et jouissent donc d'un plus grand prestige. Au niveau supérieur enfin, dominent les banquiers d'affaires (investment bankers) qui financent les offres publiques de vente (IPOs ou initial public offerings), les fusions, les acquisitions, les OPA. Généralement invisibles et simplement évoqués dans le film sous des noms imaginaires (Kahn Seidelman) ou réels (Morgan), ils constituent l'élite financière de Wall Street [6].

Les spécialistes du NYSE, placés devant leur poste de négociation (trading post), ont un rôle plus spectaculaire. Ils combinent les fonctions de courtier et de trader et endossent en outre pour la bourse des responsabilités de parquet. Ils doivent assurer un marché équitable et ordonné (keep a fair and orderly market) pour les titres dont ils ont la charge. Ils assument au nom du NYSE une charge cruciale et prestigieuse. En achetant ou en vendant des titres contre les tendances excessives, ils atténuent les fluctuations et protègent l'économie contre les krachs boursiers. Lors des séquences de manipulation des cours d'Anacot Steel [7] et de Bluestar Airlines [18], on peut les observer animer les enchères, renseigner les courtiers, s'enquérir de leurs intentions et leur livrer des titres.

En marge de ces métiers institutionnalisés, de nombreux types de spéculateurs individuels interviennent sur les marchés. Inspiré par les nombreuses OPA (LBOs ou leveraged buy-outs) des années 1980, Oliver Stone a mis l'accent sur les attaquants d'entreprises, appelés également prédateurs ou raiders. Les personnages de Gordon Gekko et de Sir Lawrence Wildman, les deux raiders concurrents du film, ont été calqués respectivement sur l'Américain Ivan Boesky (Stewart 1991: 94) et le Britannique Sir James Goldsmith (Nora 1989: 13). D'autres spéculateurs, les arbitrageurs ou arbs, qui opèrent discrètement dans le sillage des raiders, sont également évoqués [7]. À l'affût de raids potentiels, ils achètent des actions avant que l'OPA ne fasse monter les cours, pour les revendre ensuite à profit aux raiders qui en ont besoin pour acquérir l'entreprise (Nora 1989 : 116). En parasitant les OPA, les arbs tentent d'obtenir des prédateurs les meilleurs 
prix de vente possibles et méritent ainsi le nom de «maîtres-chanteurs au billet vert » ( greenmailers). Gekko agit en tant qu'arb pour contrecarrer une action de son rival Wildman, qui le traite logiquement de «two-bit pirate and greenmailer » [8] (Nora 1989 : 37).

\section{2. Évolutions historiques de la culture professionnelle}

15 L'intérêt de Wall Street ne se résume cependant pas à une juxtaposition mécanique des différents métiers de la bourse. Les professions sont également envisagées dans leur évolution et le film montre avec finesse qu'elles sont puissamment travaillées par des forces historiques profondes.

Les deux jeunes courtiers, Bud et Marvin, sont des yuppies typiques des années 1980 et ils sont manifestement rompus à l'informatique boursière. De nombreuses allusions laissent entendre qu'ils se rattachent majoritairement à l'école des chartists ou des technical analysts [4, 6]. À l'aide de logiciels sophistiqués, les chartistes tentent de déduire des graphiques boursiers (charts) les évolutions futures des cours et ils privilégient les gains spéculatifs immédiats. À l'inverse, les courtiers de l'ancienne génération, comme Lou Mannheim et Dan Steeples, sont des fondamentalistes. Mal à l'aise devant un ordinateur [4], ils s'appuient sur des données économiques de base (fundamentals [1, 7]) comme les ratios, le revenu par action, le rapport cours/bénéfices, pour prendre des décisions d'investissement à long terme. Échaudés par la Grande Dépression, ils se méfient de la spéculation à court terme (voir Lou [1]). La rivalité entre chartistes et fondamentalistes agite la communauté boursière depuis les années 1930 et a été ravivée dans les années 1980 par le développement rapide de l'informatique financière. Le film reflète cette évolution historique et la discussion entre les yuppies (Bud et Marvin) et le vieux Lou Mannheim en début de film oppose clairement les deux écoles [1]. Marvin refuse d'investir dans la société que Lou lui recommande s'il faut attendre pour qu'elle fasse des bénéfices («They'll take five years to come around »). Et Lou de lui répondre : «Stick to the fundamentals, that's how IBM and Hilton were built. Good things sometimes take time ».

er des bretelles multicolores était le signe distinctif des yuppies et le spectateur remarque que les bretelles de Bud se font de plus en plus voyantes au fur et à mesure qu'il réussit [12]. Le dénouement montre également la grande immaturité de la jeune génération qui n'avait jamais connu de crise boursière. Le krach d'octobre 1987 mit fin aux rêves des yuppies. Il suivit de quelques jours la sortie du film et en confirma la vision prophétique.

18 Une évolution profonde de même type touche le métier des courtiers. Le père de Bud, qui déteste Wall Street, traite son fils de vendeur («You're a salesman »). Bud lui répond : " Look Dad, I'm not a salesman. How many times I gotta tell you I'm an account executive " [2]. L'échange est révélateur de la différence de perception entre la génération du père et celle du fils. À la suite de la crise de 1929, Wall Street et le métier de vendeur de titres pâtirent d'une réputation désastreuse parmi le grand public américain. Le père de Bud leur en garde probablement rancœur depuis l'enfance. Afin de restaurer la confiance du public, la SEC adopta l'appellation plus respectable de registered representatives pour désigner les courtiers (brokers). Dans le même but, Charles Merrill, le fondateur de la grande firme Merrill Lynch, donna à ses vendeurs le titre d'account executives au début des années 1940. La nouvelle dénomination fut appréciée par le public et se répandit rapidement dans la communauté boursière (Perkins 1999: 175) ${ }^{9}$. En rappelant son titre officiel, Bud tente de retrouver sa dignité professionnelle aux yeux de son père, qui ne se 
laisse pourtant pas influencer (« You get on the phone and ask strangers for money, right? You're a salesman »).

Le film montre également que les métiers de la bourse subissent et transcendent les pesanteurs sociales. L'analyse dépasse ici l'étude du substrat professionnel et touche à la civilisation. Le quartier financier abonde en jeunes gens de bonne famille formés dans les universités prestigieuses de la côte est. Ils se retrouvent dans les clubs huppés exclusifs interdits au tout venant [5]. Issu d'un milieu modeste [6], Gekko n'a étudié que dans une université au rabais [5] mais il force le passage vers ces lieux de pouvoir à coups de millions de dollars et n'éprouve que du mépris pour les anciens de la Ivy League ( $« I$ bought my way in and now all these Ivy League schmucks are sucking my kneecaps. I just got on the board of the Bronx Zoo. It cost me a million » [5]). Il explique à Bud Fox que dans le monde darwinien de Wall Street, aller à Harvard ou à Yale est un handicap plutôt qu'un atout : " I've been in this business since '69. Most of these Harvard MBA types don't add up to dogshit. Give me guys that are poor, smart and hungry. " [6] La supériorité de la cupidité primitive sur la bonne éducation est magnifiquement exprimée par son discours devant les actionnaires de Teldar Paper : "The point is, ladies and gentlemen, greed is good. Greed works, greed is right. Greed clarifies, cuts through, and captures the essence of the evolutionary spirit » [12]. La célèbre citation « Greed is good » vient directement d'un discours qu'Ivan Boesky, une autre star de Wall Street, d'origine modeste comme Gekko, présenta le 18 mai 1986 devant la promotion des nouveaux diplômés de la business school de l'université de Berkeley (Stewart 1991 : 222). Gekko s'inscrit ainsi dans la longue tradition des grands opérateurs individuels qui, partis de rien, se hissent aux sommets de Wall Street à la force du poignet comme Jesse Livermore, le roi des spéculateurs au début du XXe siècle ; Muriel Siebert, la première femme membre du New York Stock Exchange en 1967 et Ivan Boesky lui-même.

\section{Les opérations boursières}

Outre les activités traditionnelles d'achat et de vente de titres, Wall Street présente des opérations spéculatives plus spectaculaires qui contribuent aux fluctuations du marché et qui donnent le statut de grand opérateur à ceux qui les initient. Nous en analysons deux qui sont au cœur de l'activité de Wall Street: la vente à découvert (bear sale) et l'OPA hostile (corporate raid).

\subsection{La vente à découvert}

21 La plupart des opérateurs boursiers essaient d'acheter leurs titres à bas prix pour les revendre plus cher. Ils sont appelés haussiers (bulls, c'est-à-dire «taureaux» dans le jargon de Wall Street) car leurs gains dépendent de la hausse des cours. Certains spéculateurs, néanmoins, profitent de la chute des cours grâce à une technique particulière, la vente à découvert. Ils sont appelés baissiers (bears, " ours »). La vente à découvert (short sale ou bear sale) est l'opération la plus classique de la spéculation boursière. Elle consiste à vendre des titres que l'on n'a pas, dans l'espoir de les racheter plus tard moins chers juste avant de les livrer. Dans un film comme Wall Street, il était presque inévitable que Gekko endosse le costume du grand spéculateur baissier. Une remarque de Marvin, aussi fugace qu'admirative, révèle ses talents de bear :

Oh, Gekko, Gekko is beautiful! Thirty seconds after the Challenger blew up, he is on the phone selling NASA stocks short! [2] 

causant la mort de sept astronautes, Gekko comprend instantanément que les actions des sociétés impliquées dans le projet de la NASA (NASA stocks) ${ }^{10}$ vont chuter. Il téléphone à son courtier pour en vendre alors qu'il n'en a pas (to sell short) ${ }^{11}$. Les cours mettant environ une à deux minutes pour réagir aux nouvelles, Gekko, en intervenant dans les trente secondes, vend ses titres avant que les prix ne commencent à chuter. Sachant que le NYSE fonctionne selon la règle $\mathrm{T} 3$ (Trade +3 days) ${ }^{12}$, Gekko pourra les racheter à bas prix avant de les livrer. Il gagne la différence entre le prix de vente élevé obtenu en T1 et le prix d'achat déprimé par la catastrophe en $\mathrm{T} 3$.

Pour les yuppies, cette opération relève de l'exploit. En effet, la vente à découvert est extrêmement risquée et, pour s'y aventurer, le spéculateur doit être persuadé que les cours vont baisser. En effet, si les cours montent (si la mission Challenger avait été un succès, par exemple), il se voit contraint de racheter les titres à un prix plus élevé que le prix de vente et il perd d'autant plus qu'il a été cupide. Ce cas de figure s'appelle bear squeeze, "l'étranglement des baissiers à découvert", parce qu'ils sont pris en tenaille entre la baisse qu'ils avaient escomptée et la hausse qui les dépouille. C'est la raison pour laquelle des batailles boursières acharnées opposent depuis toujours les bulls et les bears. L'issue en est, selon la position de chacun, la fortune ou la ruine. L'histoire de Wall Street est une longue suite de millions gagnés ou perdus à la hausse et à la baisse. Les enjeux considérables de ce type de spéculation font de la bourse le jeu suprême, the world's greatest game (Gordon 1999; Wyckoff 1985: 180) où les baissiers, comme de fascinants danseurs de corde, sont toujours en équilibre instable entre débâcle et apothéose.

C'est en ce sens que la remarque de Marvin, malgré sa brièveté, prend une importance beaucoup plus large que le simple mécanisme spéculatif qu'elle décrit. Elle évoque le cœur de l'activité professionnelle de Wall Street et elle inscrit Gekko dans la lignée des grands opérateurs à la baisse : Jacob Little, Charles Woerishoffer, Jesse Livermore, et plus près de nous George Soros dont les attaques baissières forcèrent la Banque d'Angleterre à dévaluer la livre sterling en 1992. Le cynisme égoïste de Gekko qui spécule sur les morts de Challenger et l'admiration inconditionnelle des yuppies qui seraient prêts à l'imiter si un autre drame leur en donnait l'occasion ne relèvent même pas de l'exagération propre à la fiction. Il se pourrait bien que cet épisode choquant, inventé par Oliver Stone pour stigmatiser les excès financiers des années 1980, ne soit qu'une faible anticipation d'une réalité plus inquiétante encore. En effet, à la suite des attentats du 11 septembre 2001 sur le World Trade Center, les autorités financières américaines et européennes ont relevé un nombre anormal de ventes à découvert sur des compagnies aériennes et des sociétés d'assurance. Certains intervenants auraient pu avoir vent des projets d'attentats et, exactement comme Gekko, ils auraient tenté de profiter des baisses de cours inévitablement entraînées par la catastrophe. La clôture des marchés, du 11 au 17 septembre 2001, les aurait empêchés de récupérer leurs gains, privant ainsi les enquêteurs de preuves tangibles (The Economist 2001 : 76). Ce cas particulier montre qu'un élément fictif de substrat professionnel, même s'il paraît a priori invraisemblable, peut être rendu vraisemblable par la réalité, pourvu qu'il respecte la logique professionnelle sous-jacente.

Notons enfin l'anachronisme de l'épisode : le film est daté de 1985 (date de la mort du père d'Oliver Stone), un an avant la mission Challenger. 


\subsection{Les OPA hostiles} l'activité économique. Tout autant que les ventes à découvert, elles ont contribué à l'histoire de Wall Street depuis les célèbres batailles boursières du XIX ${ }^{e}$ siècle pendant lesquelles les «barons voleurs" (robber barons) se disputaient les compagnies de chemin de fer de la Conquête de l'Ouest (Josephson 1934).

Gekko est donc un spéculateur doublé d'un raider. Sa stratégie consiste à acheter rapidement les actions d'une société sous-évaluée en bourse. Il la démantèle de façon à isoler les divisions les plus rentables, appelées « joyaux de la couronne » (crown jewels), de celles qui sont moins intéressantes. Il vend les joyaux de la couronne à un prix élevé et le reste au mieux. Si le prix total de la vente des différentes unités dépasse nettement le prix d'achat des actions, le raider gagne une fortune. La réussite d'un raid dépend donc du calcul précis de la valeur comptable d'une entreprise. La logique d'un raider boursier n'est pas industrielle, elle est exclusivement financière. Si une entreprise est surprise par un raid - elle est alors appelée «belle au bois dormant» (sleeping beauty) - elle peut faire appel à un allié financier appelé « chevalier blanc » (white knight [6]) qui l'aide à repousser l'OPA hostile du "chevalier noir » (black knight). Pour lâcher sa proie, le chevalier noir peut accepter de revendre au chevalier blanc les actions qu'il a acquises en s'efforçant de faire monter les enchères aussi haut que possible par un «chantage au billet vert» ( greenmail [8] ${ }^{13}$.

28 Les ingrédients et la terminologie des raids se retrouvent dans l'OPA que Gekko lance contre la société Teldar Paper et le repérage pédagogique du substrat professionnel est particulièrement facile. Il s'avère plus complexe en revanche pendant la première entrevue de Bud avec Gekko. Tout en discutant avec le jeune courtier, Gekko prépare plusieurs raids au téléphone et le spectateur n'en perçoit que des bribes éparses. Le script est pourtant d'une qualité telle qu'à partir de ces quelques bouts de phrases, il est possible de reconstituer le scénario des opérations. Nous donnons quelques-uns de ces passages accompagnés de leur interprétation dans la logique de raids.

Gekko : I loved it at forty. It's an insult at fifty. [3]

Interprétation : Le raid était rentable à 40 dollars l'action. À 50 dollars, la cible est trop chère. Le raid n'est plus rentable et Gekko se retire.

Gekko: Listen, Jerry, I am looking for a negative control, okay? No more than 30-35\%. Just enough to block anybody else's merger plans and find out from the inside if the books are cooked. [3]

Interprétation : Dans ce nouveau cas, Gekko ne recherche pas la majorité des actions mais une proportion qui lui donne un contrôle négatif sur la société. Il souhaite détenir de 30 à $35 \%$ des actions, et donc le même pourcentage de votes, pour empêcher les prises de décision stratégiques qui pourraient contrarier ses plans, comme par exemple une fusion (merger). Les raiders n'aiment pas que leur cible (target) se lance dans de vastes opérations pendant qu'ils préparent leur attaque. Les fusions et les acquisitions augmentent la taille de l'entreprise et donc son prix. Elles en compliquent également beaucoup l'évaluation comptable, ce qui peut compromettre le raid. En outre, détenir $30 \%$ des actions lui donnera accès à tous les comptes de l'entreprise. Il pourra vérifier s'ils ne sont pas truqués ${ }^{14}$. Sur cette affaire, Gekko en est à la phase préparatoire. 
Gekko : Let's go, guys. Looks like we are going over $5 \%$ in Teldar. Start the lawyers on a tender offer and 13D. We buy everything in sight, but don't pay over $\$ 22$. They're gonna fight, they got Myers and Thromberg doing their legal. [4]

Interprétation : Dans l'affaire Teldar Paper en revanche, Gekko est en train de lancer son raid. Il va acheter autant d'actions que possible et acquérir plus de $5 \%$ du capital. Dans ce cas, la loi exige qu'il remplisse le formulaire officiel 13D qui sera rendu public dix jours plus tard par l'autorité des marchés, la Securities and Exchange Commission (Nora 1989: 37). Il dispose donc de dix jours d'anonymat pour tenter d'acquérir la majorité des actions sans éveiller l'attention des arbs qui pourraient profiter de la hausse des cours résultant de ses achats. Il ordonne à ses collaborateurs d'acheter "tout ce qui passe", sans toutefois payer plus de 22 dollars car, au-delà, la rentabilité du raid pourrait être compromise. Il anticipe une contre-attaque juridique de la cible quand le raid sera rendu public.

Au cours de la même entrevue, le jeune Bud Fox propose des affaires juteuses à Gekko. Elles sont présentées d'une façon tout aussi elliptique mais tout aussi logique dans la perspective de raids.

Bud: Whitewood-Young Industries. Explosive earnings, great cash flow, 30\% discount from book, couple of $5 \%$ holders. [3]

Interprétation: Bud propose une entreprise intéressante à acquérir. Elle dispose de beaucoup de liquidités et coûte $30 \%$ moins cher que sa valeur comptable (book value). De plus, elle sera facile à acheter puisque seuls quelques actionnaires détiennent plus de $5 \%$ du capital. Les autres petits porteurs constituent un actionnariat éparpillé et seront heureux d'accepter ce que leur proposera le raider. Gekko n'est pas intéressé.

Bud: Terafly... Analysts don't like it. I do. The breakup value is twice the market price. This deal finances itself. You sell off two divisions, keep the core business...

[4]

4 Interprétation: Devant le premier refus de Gekko, Bud propose une perle encore plus rare, Terafly. La valeur à la casse de cette entreprise (breakup value), c'est-à-dire son prix minimum envisageable, est deux fois plus élevé que sa valeur en bourse. Dans le pire des cas, la revendre couvrira la totalité de l'achat et rapportera autant en bénéfice. Il suffit de se débarrasser de deux divisions (qu'on devine peu rentables) et de garder l'activité principale, qui constitue probablement les joyaux de la couronne.

Le sens et la logique des informations financières que les personnages échangent au cours de dialogues fiévreux échappent largement au spectateur. Celles-ci constituent cependant un maillage d'authenticité extrêmement serré et elles servent de fondation solide à la vraisemblance de l'intrigue qui s'en nourrit (Petit 1999: 68). En dernière analyse, le substrat professionnel d'une œuvre de fiction repose sur la qualité de ses éléments techniques. L'ensemble d'une culture professionnelle ne peut être correctement évoqué dans un roman ou dans un film que si ses plus petits détails sonnent vrai. La qualité d'une œuvre de FASP dépend du talent avec lequel la technique et ces détails sont harmonieusement fondus dans l'intrigue et contribuent à la satisfaction du public. Wall Street relève ce défi avec brio. Le substrat professionnel y est impeccable et, bien que parfois opaque, il n'est jamais pesant. Un dernier exemple confirmera ce jugement.

$\mathrm{Au}$ moment où Bud quitte Gekko au terme de leur première entrevue, il passe devant Natalie, la secrétaire, qui prépare une conférence téléphonique en duplex. Le spectateur l'entend dire en arrière-fond : 
Natalie: Mr Gekko, your conference call is ready. Mr Sugarman in Delaware, $\mathrm{Mr}$

Bernard in Los Angeles. Mr Jackson and Ms Rosco in London. [4] n'est pas fait pour surprendre. Qu'il puisse en avoir au Delaware est plus inattendu. Or, c'est là-même, dans ce remplissage apparemment superfétatoire de la scène, que se loge la qualité du substrat professionnel du film. En effet, dans le monde des affaires, l'État du Delaware est bien connu pour être le plus proche équivalent américain des paradis fiscaux. De grandes sociétés comme Yahoo! ou McDonald's, dont les sièges sociaux sont en Californie ou dans l'illinois, y sont enregistrées afin de bénéficier d'avantages fiscaux. L'État a largement libéralisé sa réglementation financière dans les années 1980, et le film laisse entendre que Gekko en use comme d'un tremplin vers les centres financiers délocalisés (offshore financial centers [7, 10]) comme les Îles Caïman [10]. Wall Street fourmille ainsi de détails apparemment anodins qui sont une mine de références aux différents aspects de la finance américaine.

\section{Les institutions}

Depuis toujours, les opérateurs de Wall Street cherchent à maitriser l'information financière, que ce soit pour la monopoliser ou pour la dissimuler (Van der Yeught 2003 : 3-19). Certaines de leurs pratiques, comme le fait de tirer parti d'une information confidentielle (inside information) pour spéculer, ont été interdites par la Securities and Exchange Commission en 1934. Pourtant, la hausse rapide des années 1980 encouragea de nombreux spéculateurs à prendre des libertés avec la loi et la décennie fut marquée par les arrestations de vedettes de Wall Street telles que Dennis Levine, Ivan Boesky, Martin Siegel ou Michael Milken (Stewart 1991 : 450). l'obsession de l'information qui mène à la fortune, le sentiment général d'impunité face aux exigences de la loi. "The most valuable commodity I know of is information, wouldn't you agree? » [5] dit Gekko à Bud. Bud, lui-même, dissipe les scrupules de son ami, l'avocat Roger Barnes, qui hésite à lui transmettre des informations confidentielles : «Oh, relax Roger. Everybody is doing it now » [9].

L'aspect réglementaire et déontologique de l'activité boursière constitue une part essentielle du substrat professionnel de Wall Street. L'intrigue pose le problème de l'honnêteté professionnelle et du respect de la loi. Les personnages louvoient continuellement entre les exigences de la réglementation et la cupidité, jusqu'à la chute finale. Le film s'ouvre sur un délit d'initié (insider trading) commis par Bud pour accéder à Gekko et il se termine sur l'arrestation de Bud par les agents fédéraux [18]. Pour réduire sa peine, il accepte de coopérer avec la justice (plea bargaining) et de dévoiler les activités frauduleuses de Gekko. Le scénario suit à la lettre le déroulement des procès réels de Dennis Levine, Ivan Boesky et Martin Siegel qui s'accusèrent tous mutuellement de délit d'initié. Il anticipe même la chute en 1990 de Mike Milken, dénoncé par son ex-complice Boesky (Stewart 1991 : 231-443).

41 L'institution qui représente la loi est la SEC, omniprésente dans l'arrière-fond de l'intrigue. Bud est conscient des dangers du délit d'initié. D'emblée, il fait part de ses réticences à Gekko : "I could lose my licence. If the SEC found out, I could go to jail. That's inside information, isn't it? ». [6] Ses transactions sont surveillées par StockWatch, le service de 
contrôle du NYSE [10], et Roger Barnes lui-même reçoit des appels inquiétants de la SEC [14]. Dans sa totalité, le film peut être utilisé comme une étude de cas sur la criminalité boursière et sur les institutions de surveillance des marchés.

\section{Les aspects civilisationnels}

42 L'histoire que raconte Wall Street peut se lire comme la parabole du fils prodigue (Luc, 15, 11-32). Comme le plus jeune fils de l'Évangile, Bud quitte symboliquement son père, Carl, pour entrer à Wall Street ${ }^{15}$. La rupture, consommée au milieu du film [14], est préparée dès le début lors de la scène où père et fils se retrouvent après le travail dans un bar de banlieue [2-3]. Grâce à des prises de vue alternées en champ et contrechamp, Oliver Stone oppose clairement les classes sociales : col blanc pour le fils, col bleu pour le père [2]. Leur dialogue révèle leur désaccord de fond à propos de Wall Street. Bud récite le credo des yuppies tandis que son père, un syndicaliste qui a probablement souffert de la crise de 1929, défend les valeurs traditionnelles de l'Amérique profonde.

43 À travers le père et le fils, ce sont « Main Street » et «Wall Street » qui s'affrontent. Dans ce milieu professionnel, Main Street représente ceux qui n'appartiennent pas à Wall Street, les outsiders par opposition aux insiders. L'expression désigne les investisseurs modestes, l'Amérique des petites villes, la base manufacturière et commerçante du pays. Depuis la publication du livre de William Z. Ripley, Main Street and Wall Street, en 1927, une longue tradition oppose les deux mondes et entretient entre eux une méfiance réciproque. Les Wall Streeters méprisent les classes moyennes et populaires en qui ils voient des moutons à tondre (Elias 1971). Pour les Main Streeters, Wall Street est un univers dominé par la cupidité et par la fraude qui entretient une relation parasitaire avec l'Amérique travailleuse et productive. «I told you not to get into that racket in the first place » dit Carl à son fils, «you could have been a lawyer or a doctor. If you'd stayed at Bluestar, you coulda been a supervisor now, instead of being a salesman » [2].

Comme le fils prodigue, Bud quitte donc Main Street et son père pour chercher fortune à Wall Street auprès d'un père de substitution, Gekko. Il y trouve un milieu professionnel profondément marqué par le darwinisme social (social Darwinism). Le film s'ouvre sur les portraits d'archétypes sociaux traditionnels aux États-Unis, le loser (Dan Steeples), les winners (les deux yuppies Marvin et Bud), et le has been (Lou Mannheim), mais Wall Street y prend rapidement l'aspect d'une jungle sans merci. «It's trench warfare out there, pal » dit Gekko à Bud [6]. La nature profondément darwinienne de Wall Street trouve son évocation la plus crue dans le discours de Gekko aux actionnaires de Teldar Paper, la société sur laquelle il vient de lancer une OPA [12]. Tous les thèmes du darwinisme social y sont exprimés : l'Amérique et Teldar Paper sont malades parce qu'on laisse survivre les moins adaptés ; ils doivent être éliminés par des prédateurs plus puissants. «Well, in my book, you either do it right or you get eliminated » dit Gekko. Non seulement le raider cite Boesky ( Greed is good») mais il use également de la célèbre citation du philosophe darwinien américain William Graham Sumner "survival of the unfittest» (Sumner 1963: 76-77) : "The new law of evolution, in corporate America seems to be... survival of the unfittest ». La communauté professionnelle de Wall Street se reconnait dans les analyses darwiniennes depuis leur origine. Dès 1870, Fowler, l'un des premiers grands chroniqueurs de Wall Street, établissait un parallèle entre la concurrence parmi les opérateurs et le concept darwinien de sélection naturelle (Fowler 1870 : 35-36). Depuis, Wall Street est restée largement fidèle aux analyses du darwinisme social alors qu'elles 
ont dans l'ensemble disparu de la scène américaine après la première guerre mondiale (Hofstadter 1959: 203). La multiplication des batailles boursières et des OPA hostiles pendant les années 1980 a remis le darwinisme à la mode et les conférences annuelles organisées à Hollywood par la Drexel Burnham Lambert, une banque spécialisée dans les raids boursiers, prirent le nom de «bal des prédateurs » (Predators' Ball) (Stewart 1991: 117). Les innombrables métaphores de la langue boursière utilisées dans Wall Street portent la marque de ce darwinisme social ambiant: "You got that killer instinct " dit Gekko à son jeune élève [16].

Au darwinisme cynique de Wall Street, le film oppose la franchise rugueuse et l'honnêteté quelque peu démodée de Main Street incarnée par le père de Bud. Celui-ci se réfère aux classiques de la culture américaine, comme Mark Twain, lorsqu'il appelle affectueusement son fils « Huckleberry » $[2,10]$. Il cite la Bible afin de comparer Gekko au Pharaon d'Égypte (Exode, 1, 8 [14]). Étrangement, pour le jeune Bud l'image du père ne se limite pas à Carl et à Main Street. En effet, Carl Fox se dédouble en une autre image du père qui déborde Main Street pour se prolonger dans le domaine de Wall Street. Le spectateur comprend que Bud Fox, au sein même de Wall Street, a un second inspirateur paternel qui partage les mêmes valeurs que son père réel. Il s'agit de Lou Mannheim qui représente le propre père d'oliver Stone, Louis Stone. Ce dernier fut ruiné dans les années 1970 lors de la chute de la banque Hayden Stone où il était associé (Elias 1971 : 100). De même, le film indique que Lou Mannheim a tout perdu quand sa firme a fait faillite dans les années 1970 [4]. Bud éprouve une affection filiale pour Lou [4] qui le lui rend bien et qui l'appelle «fils» [7]. Les parallèles sont nombreux entre ces deux figures du père. Comme Carl, Lou se réfère aux classiques de la culture américaine. Lorsque Bud lui annonce "Lou, I got a sure thing », il lui rétorque la célèbre réponse de Benjamin Franklin "No such thing, except death and taxes ${ }^{16}$. Lou adopte également un ton quasi biblique quand il indique à Bud la voie de la sagesse au moment de son arrestation: «Bud, I like you. Just remember something. Man looks in the abyss. There's nothing staring back at him. At that moment, man finds his character. And that is what keeps him out of the abyss » [18].

Quoiqu'appartenant aux deux mondes opposés de Main Street et de Wall Street, le père réel Carl et le père symbolique Lou se rejoignent sur des valeurs communes. Ils pensent que Wall Street est au service de l'économie, et que l'économie est au service de l'homme. Pour eux, gagner de l'argent n'est pas une fin en soi, c'est un moyen pour faire progresser la société. Wall Street ne doit pas exploiter Main Street égoïstement car les deux mondes sont solidaires et ils ont besoin l'un de l'autre. Lorsque Bud lui propose une opération juteuse, Lou se méfie et lui répond: "You are part of something here, Bud. The money you make for people creates science and research jobs. Don't sell that out » [7]. De même, Carl Fox accorde plus d'importance aux relations humaines qu'au profit. Il est responsable syndical et il défend les intérêts de "ses hommes" [14] contre les exigences déshumanisantes de l'économie. Pour lui, les différents composants de la vie sont intimement liés et la logique de l'argent est pernicieuse parce qu'elle tend à les dissocier et à leur faire perdre leur sens. Comme Lou, Carl combat l'égoïsme de Wall Street et il recherche les voies de la solidarité sociale. De Gekko, il dit: "What you see [in me] is a guy who never measured a man's success by the size of his wallet " [14]. Il comprend que les relations amoureuses de Bud et de Darien sont perverties par l'argent : " I don't go to sleep with no whore and I don't wake up with no whore. That's how I live with myself. I don't know how you do it » [14]. Après l'arrestation de Bud, il lui recommande de changer de système de valeurs et d'harmoniser réussite économique et réussite personnelle. «Stop going for the 
easy buck and produce something with your life. Create, instead of living off the buying and the selling of others » [19].

La vision du monde des deux pères de Bud trouve son sens profond dans le concept d'« enfouissement » (embeddedness) de l'historien et économiste hongrois Karl Polanyi. Dans The Great Transformation (2001 [1944]), Polanyi explique que jusqu'à la Révolution industrielle l'activité économique n'était pas autonome par rapport aux autres activités des sociétés. Au contraire, elle était enfouie (embedded) dans un ensemble complexe de relations sociales qui comprenaient la religion et la politique. Au XIX ${ }^{e}$ siècle, l'activité économique de marché est devenue prédominante et elle s'est "désenfouie» ( disembedded) des relations sociales. Le marché est devenu une institution spécifique qui impose sa logique à la société.

[...] The control of the economic system by the market is of overwhelming consequence to the whole organization of society: it means no less than the running of society as an adjunct to the market. Instead of economy being embedded in social relations, social relations are embedded in the economic system. [...] For once the economic system is organized in separate institutions, based on specific motives and conferring a special status, society must be shaped in such a manner as to allow the system to function according to its own laws. This is the meaning of the familiar assertion that a market economy can function only in a market society. (Polanyi $2001: 60)$

Dans une société de marché, les personnes deviennent des acteurs économiques qui s'efforcent de maximiser leurs gains et la substance humaine et naturelle de la société se transforme en marchandise. La conséquence de tout progrès purement économique ne peut être que la dislocation sociale et Polanyi met en garde contre un "désenfouissement » excessif de l'activité économique dû à une confiance exagérée des économistes dans l'auto-régulation des marchés.

Éclairées par l'analyse de Polanyi, les positions de Carl et de Lou d'un côté, et de Gekko de l'autre, prennent tout leur sens. Les premiers refusent la pure société de marché, ils résistent au « désenfouissement » de Wall Street par rapport au reste de l'économie et de la société américaine. Ils tiennent aux relations humaines (familiales, professionnelles, amoureuses) et aux solidarités sociales et économiques. Ils sentent obscurément que la logique de Wall Street isole les hommes, exacerbe les égoïsmes et pousse à la dislocation sociale. Gekko, en revanche, est une créature de la société de marché et il en pousse la logique jusqu'à ses extrêmes limites. Il brise toutes les solidarités sociales qui pourraient faire obstacle au profit économique. Il conseille à Bud de faire abstraction de tout sentiment humain : «And no feelings. If you need a friend, get a dog! » [6]. Lorsque Bud lui demande pourquoi il démantèle la société de son père, Gekko extirpe complètement la logique économique de la société humaine. Leur dialogue illustre parfaitement l'analyse de Polanyi.

Bud : Why do you need to wreck this company?

Gekko : Because it's wreckable, all right! I took another look. I changed my mind.

Bud : If they [the airline staff] lose their jobs, they got nowhere to go! My father has

worked there for 24 years! I gave him my word!

Gekko : It's all about bucks, kid. The rest is conversation. [15]

Nous sommes au cœur des relations entre Wall Street et Main Street. Depuis un siècle et demi, au rythme des booms économiques, des percées technologiques et des flambées spéculatives, Wall Street est régulièrement tentée de se «désenfouir» de la société américaine et de prospérer seule en dépit de toute logique économique, de toute solidarité nationale et de toute exigence du droit. Chaque fois que, par le scandale, la 
fraude ou les krachs boursiers, le contrat social américain se trouve menacé par les excès de Wall Street, des représentants de Main Street, politiciens, journalistes, historiens, juristes, réagissent et rappellent Wall Street à son devoir d'enfouissement socioéconomique. Ils dénoncent l'égoïsme des money trusts (Brandeis 1995 [1914]), l'inégalité des chances entre Wall Street et Main Street (Ripley 1927), l'hubris ${ }^{17}$ des «barons voleurs " (Josephson 1934), les fraudes des années 1920 (Pecora 1939), les privilèges du NYSE (Ney 1970 ; Elias 1971). Le Wall Street d'Oliver Stone est une œuvre de fiction, mais elle s'inscrit dans cette longue lignée de mises en garde contre les désenfouissements de Wall Street. La dimension militante du film montre que la place et le rôle de Wall Street dans la société américaine est l'objet d'un débat continu sur les valeurs tantôt concordantes, tantôt discordantes des deux mondes. Ce questionnement permanent établit une relation profonde entre le substrat professionnel de Wall Street et la civilisation américaine qui l'abrite. Bud est le fils prodigue qui quitte le monde des solidarités socio-économiques pour découvrir dans Wall Street le monde où le profit pur tente de se désenfouir de la société. Incapable de devenir un nouveau Gekko [19], il s'y perd. Comme le fils prodigue de l'Évangile, c'est en renouant avec son père qu'il renoue avec son identité [17]. Wall Street est une parabole des hésitations de l'Amérique face aux tentations de Wall Street, c'est-à-dire face à elle-même.

\section{Langue spécialisée et traduction}

51 Pour les praticiens d'une langue de spécialité, l'intérêt des films à substrat professionnel est de faire vivre la langue d'un métier en la plaçant en situation dans une intrigue. L'artifice de la fiction s'enracine dans une authenticité technique qui est précieuse pour le pédagogue et pour les étudiants. Les personnages, pris dans une histoire, constituent autant d'hommes et de femmes à comprendre et de modèles linguistiques à imiter.

La langue de Wall Street est fortement marquée par la terminologie boursière, particulièrement celle que les années 1980 ont mise à la mode (raids, insider trading, greenmail, junk bonds). À cet égard, le film peut être considéré comme un magnifique manuel d'anglais financier mis en scène dans une série d'études de cas linguistiques. Selon son niveau d'initiation, le spectateur pénètre plus ou moins bien le jargon professionnel. Les étudiants français seront néanmoins rassurés de voir que Carl Fox luimême peine à comprendre son fils quand celui-ci lui raconte sa journée en termes techniques : «Speak English, will you! » lui dit-il [2].

53 L'explication de la plupart des expressions techniques peut être trouvée dans des glossaires spécialisés (fundamentals [1], bull market [1], to sell short [2], arbs [7]). Certaines sont pourtant très pointues, telle l'abréviation « $\mathrm{DK}$ » [2] qui n'a pu être introduite dans le film que par un professionnel du NYSE familier du parquet (Petit 1999: 71). DK vient de Don't Know et désigne une transaction qu'un courtier refuse ou prétend erronée. Les deux initiales ont donné le verbe to DK qui signifie contester une transaction (Wyckoff 1973 : 75).

54 La mise en français intéressera vivement les étudiants en traduction spécialisée. La traduction est alerte et naturelle et elle enrobe élégamment les passages les plus techniques. Les strictes limites de temps imposées par le rythme de l'action et la gestuelle des personnages ont obligé les traducteurs à privilégier l'efficacité de la compréhension au détriment de l'exactitude quand elle restait opaque au spectateur français. En 
particulier, les idiosyncrasies culturelles américaines ont été transposées de façon à être parlantes dans un contexte français. C'est ainsi que le correspondant de Gekko, situé dans le quasi-paradis fiscal du Delaware (voir en section 3.2.), est placé à Chicago dans la version française [4]. De même, la SEC (Securities and Exchange Commission) devient la СОВ (Commission des Opérations de Bourse) même si cette institution française n'a pas sa place dans l'action $[4,14]$. Parfois, malgré tous leurs efforts, les traducteurs n'ont pu toujours éviter de perdre du sens. La phrase suivante de Marvin, déjà analysée en section 3.1., est rendue de façon approximative et la traduction se solde finalement par un contre-sens technique.

Gekko is beautiful. Thirty seconds after the Challenger blew up, he is on the phone selling NASA stock short. [2]

Traduction française : Gekko, il m'enchante ce mec. La navette Challenger venait à peine d'exploser qu'il vendait déjà ses NASA, avant que ça plonge. Chapeau!

Le résultat obtenu est probablement le meilleur possible compte tenu de la rapidité d'élocution de Marvin, mais il est intéressant de faire mesurer aux étudiants la perte de sens induite. "Challenger » a été légèrement surtraduit par « la navette Challenger » mais «short » a disparu. La version française présente donc Gekko comme un opérateur très rapide, soucieux de protéger les capitaux qu'il possédait déjà. Il vend ses titres avant que les cours ne chutent. Son cynisme est plutôt passif : il se limite à penser à ses intérêts avant de songer aux astronautes décédés. Dans ce cas, l'admiration des yuppies n'est que moyennement justifiée. En revanche, la version originale fait de Gekko un cynique actif. Il profite de la catastrophe, non pour protéger ses avoirs mais pour gagner une petite fortune grâce à une spéculation à la baisse (short sale). Son talent financier en sort grandi et l'admiration que lui vouent les yuppies est alors plus compréhensible, quoique bien plus révoltante pour le spectateur. Le contre-sens entraîne donc une perte de sens importante.

Les passages les plus intéressants pour les étudiants seront probablement ceux qui présentent des contre-sens manifestes, notamment lorsque la traduction française contredit la logique du substrat professionnel. Les étudiants y verront des applications pratiques de leurs cours de traduction où on leur explique que le traducteur doit parfaitement comprendre les mécanismes techniques mis en œuvre pour traduire correctement la langue qui les explique. Deux exemples illustreront ce point.

Lors de leur entrevue initiale, quand Bud présente des projets de raid à Gekko, il décrit le premier comme suit :

[...] Whitewood-Young Industries. Explosive earnings. A 30\% discount from book. Great cash flow. Couple of 5\% holders. [3]

Traduction française: Whitewood-Young Industries. Ça explose à la cote et ça grimpe encore. Des actifs en hausse de $30 \%$. Un bon cash flow. Les actionnaires sont...

Le contre-sens porte sur « $30 \%$ discount from book». L'expression anglaise signifie que les actions de la société Whitewood-Young Industries valent $30 \%$ de moins que la valeur comptable de la société (book value). Autrement dit, l'entreprise est intéressante à acheter parce qu'elle est bon marché en bourse et qu'on pourra la revendre à profit. La traduction " Ça explose à la cote et ça grimpe encore » laisse entendre au contraire que l'action est en train de flamber. Cette information ruine le raid proposé par Bud et la traduction se révèle donc absurde. Notons que le sous-titrage français correspondant («Le titre sera à $30 \%$ de sa valeur comptable ») est logiquement cohérent mais arithmétiquement faux. Il est logique en effet que l'action soit moins chère que la valeur comptable de l'entreprise. Mais la décote (discount) étant de $30 \%$, la valeur de l'action représente $70 \%$ de la valeur 
comptable, et non $30 \%$. La traduction devrait donc être: "Les bénéfices explosent. L'action est sous-évaluée de $30 \%$ ».

Voici un autre exemple de contre-sens technique. Lors de l'entrevue Gekko-Larry Wildman, Wildman propose à son rival de lui racheter ses actions Anacot Street à 65 dollars l'une. Gekko, qui acceptera finalement 71,50 dollars, demande à Bud quel est le cours du titre.

Gekko : Buddy, what's the fair price for that stock?

Bud : The breakup value is higher. It's worth 80. [8]

Traduction française :

Gekko : Bud, tu peux donner un prix raisonnable pour ces actions?

Bud : Ça valait plus à la clôture. Ça vaut 80.

Le contresens porte ici sur breakup value. L'expression anglaise signifie "valeur de liquidation » et fait référence à la valeur minimale d'une entreprise vendue à la casse. La traduction française fait de breakup la clôture du marché qui se dit en fait close, closing time, tandis que le cours de clôture est le closing price. Le résultat est totalement absurde car il serait incompréhensible que Gekko n'accepte que 71,50 dollars de Wildman s'il peut obtenir 80 dollars en vendant ses titres sur le marché. En fait, Bud explique que la valeur minimale de l'entreprise atteignant 80 dollars, Gekko peut exiger de Wildman beaucoup plus que 65 dollars. C'est la raison pour laquelle ils s'entendent finalement sur le prix intermédiaire de 71,50 dollars. La traduction devrait donc être : « La valeur de liquidation est plus élevée [que les 65 dollars proposés par Wildman]. L'action vaut environ 80 ».

Dans Wall Street, les graves erreurs de traduction sont peu nombreuses et sans incidence sur la réalité puisque nous sommes dans une œuvre de fiction. Les solutions retenues par les traducteurs du film en français sont de type «impressionnistes": elles visent à donner au spectateur un certain type d'impression qui lui permette de suivre l'action, même s'il n'en saisit pas précisément les détails. Une trop grande rigueur dans la recherche de l'exactitude aurait peut-être nuit au spectacle en rendant les mécanismes boursiers incompréhensibles. Toutefois, les étudiants ne doivent pas oublier que dans FASP, il y a «substrat professionnel » et que dans la réalité de Wall Street, la moindre erreur de compréhension linguistique peut coûter des milliers de dollars.

\section{Conclusion}

Dans le Wall Street d'oliver Stone, le substrat professionnel est si dense qu'il peut donner lieu à de multiples stratégies pédagogiques. Chacune exigera pourtant que le pédagogue détermine précisément la facette du film qu'il souhaite faire étudier et le degré de technicité que ses étudiants peuvent atteindre. Quelle que soit l'approche adoptée, il nous paraît préférable d'utiliser le film sur une période prolongée. En effet, c'est sa cohérence interne, beaucoup plus que la juxtaposition de ses différentes facettes, qui donne accès à la cohérence du monde américain de la bourse.

Avec des étudiants de gestion ou d'économie et de finance de niveau Bac +2 à Bac +4 , le film peut être utilisé comme un document professionnel d'initiation générale à la finance américaine et à la bourse de New York : décors, marchés, opérateurs, ambiance, enjeux, action, langue spécialisée peu technique.

64 Avec des étudiants plus pointus en banque-finance-bourse de niveau $\mathrm{Bac}+4 / \mathrm{Bac}+5$, le professeur peut entrer dans le détail des opérations boursières, le jargon, les institutions. 
Une exploitation alternée exposés/film peut être envisagée. Des étudiants présentent des exposés théoriques sur la vente à découvert, le délit d'initié, la SEC, les raids, la hausse boursière des années 1980, les vedettes de la finance (Boesky, Milken), la langue technique, et ils les illustrent grâce à des passages du film. Des spécialistes de la traduction peuvent comparer les versions anglaise et française et élaborer des lexiques permettant de comprendre les mécanismes présentés.

Des étudiants de civilisation peuvent également analyser des différentes facettes de la société américaine mises en scène : Main Street contre Wall Street, le darwinisme social, les réseaux métaphoriques tissés dans la langue. Les historiens de l'économie peuvent en tirer d'intéressantes comparaisons entre les années 1920, les années 1980, les années 1990, trois périodes de hausses boursières qui se sont conclues par des krachs historiques. Il va sans dire que toute exploitation pédagogique se prête à d'innombrables débats entre les étudiants et le professeur. Wall Street est un film qui a la chance d'être perpétuellement rejoué et renouvelé dans la réalité. Il suffit de consulter les indices boursiers et de suivre l'actualité financière pour constater que la réalité illustre la fiction tout autant que la fiction illustre la réalité. C'est le plus grand mérite d'une FASP réussie.

\section{BIBLIOGRAPHIE}

Brandeis, Louis D. 1995 [1914]. Other People's Money and How the Bankers Use It. New York : Bedford Books.

Cohen, J.M. \& M.J. Cohen. 1992. The New Penguin Dictionary of Quotations. Londres : Penguin.

Economist (The). 29 septembre 2001, $\mathrm{n}^{\circ} 8241$.

Elias, Christopher. 1971. Fleecing the Lambs. Chicago : Henry Regnery Company.

Fowler, William Worthington. 1870. Ten Years in Wall Street, or Revelations of Inside Life and Experience on Change. Hartford, Connecticut : Worthington, Dustin \& Co.

Gordon, John Steele. 1999. The Great Game: A History of Wall Street. Londres : Orion Business Books. Hofstadter, Richard. 1959 [1944]. Social Darwinism in American Thought. New York : George Braziller, Inc.

Josephson, Matthew. 1934. The Robber Barons: The Great American Capitalists. New York : Harcourt Brace.

Lefevre, Edwin. 1994 [1923]. Reminiscences of a Stock Operator. New York : John Wiley \& Sons. Ney, Richard. 1970. The Wall Street Jungle. New York : Grove Press.

Nora, Dominique. 1989. Les possédés de Wall Street. Paris : Gallimard, Folio/Actuel n¹7.

Pecora, Ferdinand. 1939. Wall Street under Oath. New York : Simon \& Schuster.

Perkins, Edwin J. 1999. Wall Street to Main Street. Cambridge : Cambridge University Press.

Petit, Michel. 1999. « La fiction à substrat professionnel : une autre voie d'accès à l'anglais de spécialité ». ASp 23-26, 57-81. 
Polanyi, Karl. 2001 [1944]. The Great Transformation. Boston : Beacon Press.

Ripley, William Z. 1927. Main Street and Wall Street. Boston : Little, Brown and Co.

Stewart, James B. 1991. Den of Thieves. New York : Simon \& Schuster.

Sumner, William Graham. 1963. Social Darwinism: Selected Essays of William Graham Sumner.

Englewood Cliffs, New Jersey : Prentice Hall.

Van der Yeught, Michel. 2003. « Le transfert d'information, moteur de l'histoire boursière

américaine ». ASp 39-40, 3-19.

Wyckoff, Peter. 1973. The Language of Wall Street. New York : Hopkinson \& Blake.

Wyckoff, Richard D. 1985 [1930]. Wall Street Ventures \& Adventures Through Forty Years. Burlington, Vermont : Frazer Publishing.

\section{ANNEXES}

\section{Le script de Wall Street}

Le script de Wall Street peut être téléchargé sur Internet à partir des deux sites suivants. $<$ http://www.dailyscript.com/scripts/wall_street.html> (2004).

Ce premier site fournit le script original écrit en avril 1987 par Stanley Weiser et Oliver Stone. Il tient sur 91 pages et comporte des indications précises sur la mise en scène, le jeu et la psychologie des personnages. En tant qu'archive, cette version est très intéressante car elle permet de comprendre le projet initial des scénaristes et les intentions du réalisateur. En revanche, elle ne permet qu'un suivi approximatif du scénario final. De nombreux passages ont été omis de la version filmée et, dans les morceaux retenus, les écarts entre le script et les dialogues réels sont fréquents.

$<$ http://www.bunri-c.ac.jp/ suzuki/movie/hints/wallst3.txt> (2004).

Ce second site fournit une version brute du script sur 19 pages. Malgré de nombreuses approximations orthographiques, le texte suit très fidèlement les dialogues réels, mais ne comporte aucune indication sur les personnages ou la mise en scène. Les répliques se succédant sans qu'on sache qui les prononce, il est préférable d'utiliser cette version tout en visionnant le film en parallèle. Pour des raisons de précision et de commodité, c'est à la pagination de ce script que renvoient les numéros entre crochets dans cet article.

\section{Filmographie}

Boiler Room (Les Initiés)

Réalisateur : Ben Younger. Acteurs : Giovanni Ribisi, Vin Diesel, Nia Long, Nicky Katt, Scott Caan, Ben Affleck. New Line Productions, 1999. Version DVD par Metropolitan Film.

Rogue Trader (Trader)

Acteurs : Ewan McGreggor, Anna Friel. Granada Fims, 1998. Version DVD par Capitol Film.

Wall Street

Réalisateur : Oliver Stone. Acteurs : Michael Douglas, Charlie Sheen, Martin Sheen, Daryl Hannah, Terence Stamp. 20th Century Fox, 1987. Version DVD par 20th Century Fox Home Entertainment. 


\section{NOTES}

1. Les titres originaux respectifs sont Rogue Trader et Boiler Room.

2. Michael Douglas a obtenu un Academy Award pour son interprétation de Gordon Gekko.

3. Voir le film américain Les Initiés (Boiler Room), déjà cité, dans lequel une scène montre le gang des jeunes vendeurs de titres spéculatifs en train de regarder des passages de Wall Street et d'en réciter les dialogues qu'ils connaissent par cœur.

4. Yuppie vient des trois lettres $\mathrm{y}, \mathrm{u}, \mathrm{p}$, qui, selon les interprétations, signifient young urban professional ou young upwardly-mobile professional. Professional a évidemment le sens anglais de profession libérale, ce qui inclut les avocats, les comptables et les courtiers.

5. Le script de Wall Street est disponible sous deux formes distinctes. Le script original complet, assez différent des dialogues réels et le script réel qui reproduit assez précisément les dialogues. Pour plus de commodité, les références entre crochets renvoient aux pages du script réel téléchargeable sur le site Internet suivant: <http://www.bunri-c.ac.jp/ suzuki/movie/hints/ wallst3.txt>. En cas de divergences avec les dialogues entendus à l'écran, les citations fournies correspondent toujours aux dialogues du film. Ce script comportant dix-neuf pages, les numéros de pages entrecrochets indiquent approximativement les passages du film où se situent les scènes et les dialogues cités.

6. La décimalisation des cours est achevée depuis 2001.

7. Cette information est fournie dans le script original page 35. Le site Internet où ce script est disponible est indiqué en bibliographie.

8. WASP : White Anglo-Saxon Protestant.

9. La traduction de la version française du film, qui ne peut faire ressortir la nuance entre account executive et broker/salesman, garde " courtier".

10. Les étudiants pensent souvent que NASA stocks désigne les actions de la NASA. L'agence étant gouvernementale et financée par le Congrès sur proposition du président, elle n'est pas une société cotée et elle n'émet ni actions ni obligations. NASA stocks désigne donc les valeurs du secteur aérospatial dans son ensemble.

11. Avoir des titres se dit to be long, ne pas les avoir se dit to be short. Vendre à découvert, c'est-àdire sans avoir les titres, se dit to sell short.

12. Règle $\mathrm{T} 3$ (Trade +3 days) : le paiement et la livraison des titres doivent être effectués au maximum trois jours après la transaction. Pendant cette période, les cours peuvent fluctuer.

13. L'ouvrage de D. Nora (1989) explique en détail le mécanisme des OPA hostiles, précise la terminologie et analyse des raids célèbres des années 1980 .

14. To cook the books signifie maquiller les comptes.

15. Notons que Carl mentionne un frère de Bud [3], qui contrairement à lui, ne semble pas lui emprunter de l'argent : serait-ce le fils aîné vertueux mentionné en Luc 15, 29 ?

16. "In this world nothing can be said to be certain, except death and taxes." Benjamin Franklin, Lettre à Jean-Baptiste Leroy, 13 novembre 1789 (Cohen \& Cohen 1992 : 168).

17. Orgueil démesuré. 


\section{RÉSUMÉS}

Parmi les œuvres de fiction à substrat professionnel (FASP) en anglais financier, Wall Street, le film d'oliver Stone, reste un document très utile. Bien qu'ayant été tourné en 1987, son riche substrat professionnel est toujours d'actualité. Il permet d'initier des étudiants de niveaux divers à l'anglais spécialisé et au monde professionnel des marchés financiers américains. Toute démarche pédagogique dépendant étroitement du contenu d'enseignement, cet article propose une analyse du substrat professionnel de Wall Street. Six facettes du monde de Wall Street présentées par le film sont étudiées: les lieux, les métiers, les opérations boursières, les institutions, les aspects civilisationnels, la langue et la traduction en français.

Among the works of professionally-based fiction in financial English, Wall Street, a film directed by Oliver Stone, remains a very useful document. The film was shot in 1987, but its rich professional content is still highly relevant today. It may be used to introduce different types of students to financial English and to the world of the U.S. financial markets. Because teaching approaches are largely content-dependent, this article presents a survey of Wall Street's professional content. Six distinct facets of Wall Street are examined: locations, jobs, stock market operations, institutions, civilization, language and translation.

\section{INDEX}

Mots-clés : anglais de spécialité, FASP, New York Stock Exchange, Stone (Oliver), substrat professionnel, Wall Street

Keywords : ESP, professional substratum, professionally-based fiction

\section{AUTEUR}

\section{MICHEL VAN DER YEUGHT}

Michel Van der Yeught est maître de conférences au département GEA de l'IUT de Toulon. Son sujet de recherche est Wall Street (langue, histoire et civilisation) ; il est l'auteur de L'initiation à l'anglais comptable et à la comptabilité anglo-saxonne (Ellipses 1992) ainsi que de Initiation à l'anglais financier et à la finance anglo-saxonne (Ellipses 2002). mvdy@free.fr 